\title{
Éducation et mondialisation en Tunisie
}

Education and globalisation in Tunisia

Educación y mundialización

\section{Ahmed Chabchoub}

\section{OpenEdition}

Journals

Édition électronique

URL : http://journals.openedition.org/ries/2617

DOI : $10.4000 /$ ries.2617

ISSN : 2261-4265

Éditeur

Centre international d'études pédagogiques

Édition imprimée

Date de publication : 1 décembre 1999

Pagination : 89-94

ISSN : 1254-4590

Référence électronique

Ahmed Chabchoub, «Éducation et mondialisation en Tunisie », Revue internationale d'éducation de Sèvres [En ligne], 24 | 1999, mis en ligne le 23 novembre 2012, consulté le 19 avril 2019. URL : http:// journals.openedition.org/ries/2617; DOI : 10.4000/ries.2617 


\title{
Éducation et mondialisation en Tunisie
}

\author{
Ahmed Chabchoub \\ Directeur adjoint de l'ISEFC à l'université de Tunis I
}

"Avec la mondialisation, nous sommes entrés dans une ère de transculturalité avec ses risques et ses périls, mais aussi ses chances à saisir."

Federico Mayor, 1998

La modernisation de la société tunisienne connaît, avec le phénomène actuel de mondialisation, une forte accélération que la proximité géographique avec l'Europe et le développement vertigineux des moyens d'information et de communication rendent d'autant plus inéluctable. Économique et politique au départ, ce processus de mondialisation débouche inévitablement sur le culturel puisque les réseaux ainsi créés vont véhiculer les patterns du nouvel ordre mondial, arrosant ainsi toute la planète de nouvelles valeurs.

Par ailleurs, ce nouvel ordre mondial va mettre en concurrence directe les économies avancées du Nord avec celles plus fragiles du Sud. Pour un pays comme la Tunisie, il s'agit d'un grand défi : comment se doter rapidement des moyens devant lui permettre de résister à cette confrontation pacifique ?

Quel rôle l'éducation peut-elle jouer dans ce challenge?

Porteuse de tout projet de société, l'éducation n'est-elle pas appelée à accompagner ces transformations culturelles en les expliquant aux jeunes et en amenant ces derniers à y adhérer, sans nier pour autant leurs valeurs fondamentales ? L'éducation n'est-elle pas également appelée à apprendre à ces mêmes jeunes à être performants pour pouvoir s'insérer dans ce nouvel ordre?

\section{Mondialisation et mutations culturelles}

Initiée par la révolution informatique des deux dernières décennies, la mondialisation devrait raccourcir l'espace et le temps de l'homme contemporain, rendre les frontières virtuelles et faire de la planète un grand Village, selon l'expression consacrée. Qu'il s'agisse de moyens de communication (avion, TGV... ) ou de télécommunications (télévision par satellite, fax, visioconférence, internet, GSM...), tout est aujourd'hui mis en œuvre pour raccourcir les dis- 
tances et les délais. Une technologie comme internet permet actuellement à tout abonné d'entrer en contact avec n'importe quel autre abonné (quel que soit son emplacement sur la planète) en un temps record et de lui transmettre des informations qui, en d'autres temps, auraient mis des semaines pour parvenir à son destinataire. Les événements politiques, sociaux aussi bien que les guerres et les catastrophes naturelles sont captés par les chaînes de télévision et retransmis par satellite à tous les habitants du grand Village, en temps réel. Mieux encore, cette maîtrise du temps et de l'espace s'accompagne d'une diminution des coûts qui rend l'utilisation de ces nouvelles technologies encore plus attrayante, parce que compétitive.

La généralisation progressive de ces nouveaux moyens de communication à tous les habitants de la planète va créer de nouvelles courroies de transmission des valeurs et des modèles, courroies d'autant plus efficaces qu'elles sont rapides et attrayantes (pensons aux images numériques de la télévision). C'est ainsi que de nouveaux patterns vestimentaires, musicaux, architecturaux, culinaires, esthétiques vont pouvoir quitter leur lieu d'origine pour nomadiser à travers le grand Village planétaire, offrant aux plus fragiles (les adolescents, les démunis), des objets de fixation et de désir. Les nouvelles connaissances scientifiques, les récentes découvertes technologiques vont également emprunter les mêmes canaux de transmission pour venir arroser nos sociétés et bousculer nos certitudes.

Cette circulation des idées et des valeurs est actuellement à sens unique (c'est-à-dire du Nord vers le Sud, pour les raisons que nous connaissons tous), ce qui risque de rendre la circulation de l'information (élaborée dans le Nord) quelque peu agressive et de donner à la mondialisation, aux yeux de certains, un relent de néocolonialisme.

Mais quel que soit notre jugement de valeur, cette nouvelle donne va accélérer, on s'en doute, le processus d'acculturation des sociétés maghrébines, processus commencé lentement au début du Xxe siècle. Or, à peine commençonsnous, grâce à l'éducation massive des jeunes notamment, à assimiler les valeurs de la modernité, qu'on nous parle déjà du passage urgent vers la postmodernité, ce nouvel avatar de la mondialisation. Or, ce passage rapide d'un modèle culturel endogène vers un modèle culturel exogène est de nature à produire des interférences d'autant plus difficiles à gérer par nos concitoyens (qui ont trois mille ans d'histoire derrière eux) que nos sociétés n'ont pas eu le temps d'inventer et de mettre en place de nouveaux mécanismes de transition.

Et c'est là que l'éducation peut intervenir pour expliquer ce phénomène complexe aux jeunes, les convaincre d'y prendre une part active et leur donner par-là même les outils intellectuels pour créer des passerelles entre le local et l'universel. 


\section{Apprentissage des outils de la mondialisation}

La première leçon (argumentée) que l'école doit apprendre aux jeunes, à propos de la mondialisation, est qu'il est de l'intérêt de tous de s'y inscrire, à la fois pour être dans le sens de l'histoire mais aussi pour préserver l'avenir. Se soustraire à cette dynamique globale, sous prétexte qu'elle est acculturante, est un acte suicidaire pour tous. L'histoire récente a montré que nos sociétés n'évoluent que par acculturation ${ }^{1}$ et que toute velléité de fermeture culturelle conduit à terme vers une régression sociale et culturelle.

Mais il ne suffit pas de faire ces déclarations solennelles pour s'inscrire dans la mondialisation. Encore faudrait-il se donner les moyens opérationnels pour y participer. Ces moyens sont de trois sortes : communicationnels, technologiques et éthiques.

\section{Les moyens de communication}

Pour s'inscrire dans la mondialisation, il faut dialoguer avec les habitants du grand Village, échanger avec eux biens matériels et valeurs culturelles. Plus que jamais, l'apprentissage des langues étrangères devient ici une nécessité d'adaptation au contexte global de la mondialisation. Pour communiquer avec les autres habitants du village cosmopolite, il faut maintenant connaître trois ou quatre langues.

Le Tunisien n'a pas à faire de complexes vis-à-vis des langues, puisqu'il a toujours appris celles des autres, au gré des configurations politiques et culturelles du monde ${ }^{2}$. C'est ainsi que Marrou ${ }^{3}$ rapporte que l'Africain scolarisé au IVe siècle parlait trois langues : sa langue maternelle (punique ou berbère), le latin et le grec.

C'est dire que nous ne pouvons gagner le pari de la mondialisation qu'avec plus d'éducation devant permettre d'apprendre les langues étrangères à un plus grand nombre de nos concitoyens.

1 Rappelons à cet effet le combat de la modernisation au début de ce siècle et les clivages qu'il a engendrés chez les intellectuels de l'époque. L'histoire a donné raison à ceux qui ont choisi la modernité.

2 Durant son histoire trois fois millénaire, le Maghrébin a non seulement appris les langues des autres (latin, arabe, français) mais il y a souvent excellé. Deux exemples pris à deux moments de l'histoire maghrébine : saint Augustin qui, au ve siècle a été un grand écrivain de la latinité ; et les auteurs maghrébins francophones contemporains (de Memmi à Kateb Yassine en passant par Tahar Ben Jalloun et Driss Cheraibi).

3 Histoire de l'éducation pendant l'Antiquité, Seuil, 1955. 


\section{L'apprentissage \\ des nouvelles technologies}

On ne peut vouloir être citoyen du monde et surseoir à l'apprentissage des nouvelles technologies, en premier lieu, l'usage de l'ordinateur. L'ordinateur devrait aujourd'hui avoir sa place à l'école autant que le livre. C'est tout à la fois un outil d'apprentissage, un mode de communication et la clé sans laquelle on ne peut maîtriser les nouvelles technologies de l'information.

L'usage d'internet à l'école, avec les mille et une possibilités de dialogue et d'instruction qu'il offre aux élèves, devrait lui aussi être généralisé à toutes nos écoles, comme c'est le cas en Europe et en Amérique. Par ailleurs, nous sommes convaincus que l'apprentissage de ces technologies à l'école (avec la dimension humaine qui accompagne toute œuvre éducative) leur donne une dimension culturelle et humaniste qu'elles ne sauraient avoir si elles étaient acquises ailleurs. La présence de l'éducateur, comme accompagnateur averti tout autant de leurs valeurs que de leurs limites, leur assure un usage à la fois modéré et adapté aux circonstances.

\section{L'apprentissage de la nouvelle éthique}

Apprendre à vivre dans le grand Village planétaire, cosmopolite et multiculturel, requiert des valeurs humanistes et universelles dont l'enseignement et la diffusion reviennent encore une fois à l'école.

La tolérance (c'est-à-dire l'acceptation de l'autre comme différent de soi), l'ouverture à l'autre (c'est-à-dire la connaissance de sa culture et de ses valeurs), la convivialité (c'est-à-dire la capacité de vivre et de travailler avec l'autre même s'il est différent) constituent l'ossature de la nouvelle éthique universelle qu'impose la mondialisation à tous les citoyens du grand Village ${ }^{4}$.

Là aussi, il ne s'agit pas d'endoctriner les jeunes en leur imposant cette nouvelle éthique, au nom d'une certaine mondialisation triomphante. Il s'agit de les convaincre des changements culturels en œuvre un peu partout dans le monde et de l'intérêt que nous avons tous à nous y inscrire, en toute connaissance de cause et avec toute la modération qu'impose la sagesse séculaire du Tunisien.

L'école peut ainsi prendre appui sur l'histoire pour convaincre les élèves que pour survivre et évoluer, les sociétés n'ont pas intérêt à prendre le contresens de l'histoire. La géographie, en expliquant les proximités, peut également prendre une part active à cette œuvre de conscientisation des jeunes.

4 En fait, on ne fait que revenir à la morale universelle de la Déclaration des droits de l'homme et du citoyen, Paris, 1789. 
Ici plus que partout ailleurs, le rôle de l'école reste irremplaçable : en expliquant méthodiquement les enjeux socioculturels, en démontrant rationnellement les choix et en argumentant logiquement les débats, l'école est l'institution la mieux outillée pour créer cet homme nouveau et lui apprendre à vivre dans le grand Village et à prendre part à ses activités.

\section{Éducation universelle et sauvegarde de la personnalité tunisienne}

Venons-en maintenant aux retombées culturelles de ce processus de mondialisation. La personnalité tunisienne (à peine sortie du modèle traditionnel) subit de plein fouet les patterns triomphants de la mondialisation.

Étant donné la souplesse de leurs structures mentales et affectives, les jeunes sont généralement plus perméables à ce phénomène que les autres. C'est ce qui nous autorise à parler ici de processus d'acculturation (Camilleri, 1998). Ce processus risque à terme, si l'éducation n'y prend garde, de déstructurer la personnalité de base des Tunisiens.

Comment l'éducation peut-elle gérer au mieux ce processus complexe? Comment peut-elle tirer profit du phénomène de globalisation, tout en sauvegardant l'essentiel ? Terrible paradoxe! Mais l'éducation, en tant qu'action qui s'adresse à l'être humain, complexe et multidimensionnel, n'est-elle pas dans son essence, gestion des paradoxes.

L'histoire de l'éducation montre que ce paradoxe peut être géré pédagogiquement et cela, en deux temps.

Premièrement, apprendre aux jeunes, à l'école (en cours d'éducation civique par exemple), que la vie sociale n'étant pas faite seulement d'instrumentalités, tout citoyen ne peut garantir sa pérennité qu'en s'inscrivant dans une culture locale, porteuse de valeurs. Or, certains éléments de la culture tunisienne traditionnelle (la famille, le respect des personnes âgées, etc.) peuvent être retenus et valorisés en tant qu'antidote à la dépersonnalisation sécrétée par la société postmoderne. Dans le même sillage, l'école devrait également convaincre les jeunes que l'histoire, vivier des valeurs, fonctionne pour chaque individu comme source d'enracinement dans une communauté culturelle. C'est dire qu'il ne nous suffit pas d'enseigner l'histoire nationale aux élèves ; encore faut-il leur apprendre comment s'y ressourcer.

L'école peut également persuader les jeunes Tunisiens qu'on peut tout à la fois être citoyen du monde, utiliser les nouvelles technologies de l'information, parler trois ou quatre langues étrangères et revendiquer sa culture originelle.

5 D'après Marguerite Mas, le rôle essentiel de l'éducation est de gérer les paradoxes et les contradictions : socialisation et épanouissement de l'individu, respecter l'élève et transformer son comportement, etc. 
Sans aller jusqu'à l'exemple typique du Japon, nous pouvons citer le cas de la génération tunisienne des 50/60 ans qui a su accéder à la modernité tout en gardant des liens privilégiés avec sa culture originelle. Encore une fois l'école a une tâche de conviction et non de conversion. C'est à ce prix qu'on peut assurer à cette opération succès et pérennité.

Dans un deuxième temps, on pourrait apprendre aux jeunes Tunisiens les méthodologies devant leur permettre de faire connaitre leur culture et leurs valeurs aux autres habitants du grand Village et ce, dans un double objectif : enrichir la culture générale du vis-à-vis et lui donner l'opportunité d'y trouver une solution aux effets secondaires de la postmodernité. La maitrise des nouvelles technologies de communication à l'école (internet, fabrication de cédéroms, etc.) devrait permettre aux Tunisiens de pouvoir "vendre " leur culture aux autres jeunes du grand Village. Pour ce faire, l'instrumentalité ne suffit pas; encore faut-il y croire. Croire à sa culture et en être fier (sans narcissisme, ni fanatisme) est l'œuvre de l'école et des éducateurs tunisiens. Et ils ont démontré par le passé qu'ils en étaient capables.

Qu'est ce qu'être Tunisien au xxie siècle?

Cela consiste tout d'abord à se mettre dans le sens de l'histoire et à savoir s'adapter au nouveau contexte de la mondialisation, tout en sauvegardant l'essentiel.

L'histoire récente montre que le citoyen tunisien a toujours su s'adapter aux nouvelles conjonctures et en tirer profit, tout en restant fidèle à sa personnalité de base. Que dire lorsque l'éducation, devenue depuis peu à la portée de tous les jeunes citoyens, met à la disposition de ces derniers les moyens techniques et humains devant les aider à évoluer intelligemment dans ce nouvel environnement, qui recèle pour le Tunisien autant de risques que de chances.

\section{Bibliographie}

A. Chabchoub, École et modernité en Tunisie, à parâttre.

A. Chabchoub, Nouvelle approche de l'école et de l'éducation, Tunis, 1998, (en arabe). 